\title{
On the Integrability of Classical Spinor Models in Two-Dimensional Space-Time
}

\author{
V. E. Zakharov and A. V. Mikhailov \\ L. D. Landau Institute of Theoretical Physics, Academy of Sciences of USSR, Moscow V-334, USSR
}

\begin{abstract}
Well known classical spinor relativistic-invariant two-dimensional field theory models, including the Gross-Neveu, Vaks-Larkin-Nambu-JonaLasinio and some other models, are shown to be integrable by means of the inverse scattering problem method. These models are shown to be naturally connected with the principal chiral fields on the symplectic, unitary and orthogonal Lie groups. The respective technique for construction of the soliton-like solutions is developed.
\end{abstract}

\section{Introduction}

Classical spinor systems (classical analogs of fermion fields with $c$-number values) have often been considered in the physical literature. First of all, there are the models of Nambu and Jona-Lasinio [1] and Vaks and Larkin [2]:

$$
\begin{aligned}
& \partial_{\eta} \varphi^{\alpha}=i / 2 \psi^{\alpha} \sum_{\beta} \psi^{* \beta} \varphi^{\beta} \\
& \partial_{\xi} \psi^{\alpha}=i / 2 \varphi^{\alpha} \sum_{\beta} \varphi^{* \beta} \psi^{\beta}
\end{aligned}
$$

and the Gross-Neveu model [3]:

$$
\begin{aligned}
& \partial_{\eta} \varphi^{\alpha}=-i \psi^{\alpha} \sum_{\beta}\left(\psi^{* \beta} \varphi^{\beta}+\varphi^{* \beta} \psi^{\beta}\right) \\
& \partial_{\xi} \psi^{\alpha}=-i \varphi^{\alpha} \sum_{\beta}\left(\varphi^{* \beta} \psi^{\beta}+\varphi^{\beta} \psi^{* \beta}\right),
\end{aligned}
$$

where $\eta=t+x, \xi=t-x$.

Models (1), (2) are relativistically invariant and represent systems of $N$ massless Dirac equations in a two-dimensional space-time with nonlinear (cubic) terms. Models (1), (2) correspond to the actions

$$
\begin{aligned}
& S=\int d t d x\left[\sum_{\alpha}\left(i \varphi^{* \alpha} \partial_{\eta} \varphi^{\alpha}+i \psi^{* \alpha} \partial_{\xi} \psi^{\alpha}\right)-\frac{1}{2}\left|\sum_{\alpha} \psi^{* \alpha} \varphi^{\alpha}\right|^{2}\right] \\
& S=\int d t d x\left[\sum_{\alpha}\left(i \varphi^{* \alpha} \partial_{\eta} \varphi^{\alpha}+i \psi^{* \alpha} \partial_{\xi} \psi^{\alpha}\right)-\frac{1}{2}\left(\sum_{\alpha}\left(\psi^{* \alpha} \varphi^{\alpha}+\psi^{\alpha} \varphi^{* \alpha}\right)\right)^{2}\right] .
\end{aligned}
$$


Such models arise in solid state physics as a result of the Hartree-Fock approximation to real fermion systems. They may acquire a direct quantummechanical sense as well (if the index $\alpha$ is isotopic).

Neveu and Papanicolaou [4] have conjectured that models (1), (2) can be integrated by the inverse scattering method. They [4] have succeeded in proving this hypothesis for $N=1,2$; when $N>2$ they have found that the systems (1), (2) have infinite series of conservation laws.

In the present paper systems (1), (2) are shown to be integrable for any $N$. These systems are closely connected to integrable systems previously considered principal chiral fields on Lie groups, they are in a sense the simplest versions of the latter. Model (1) is connected with a chiral field on the unitary group $\mathrm{SU}(N)$, and model (2) with a chiral field on the real symplectic group $\operatorname{Sp}(2 N, \mathbb{R})$. We consider also a new classical spinor model with the Lagrangian:

$$
\begin{aligned}
S= & \int d t d x\left[\sum_{\alpha}\left(i \varphi^{* \alpha} \partial_{\eta} \varphi^{\alpha}+i \psi^{* \alpha} \partial_{\xi} \psi^{\alpha}\right)\right. \\
& \left.-\frac{1}{2} \sum_{\alpha, \beta}\left(\varphi^{* \alpha} \varphi^{\beta}-\varphi^{* \beta} \varphi^{\alpha}\right)\left(\psi^{* \alpha} \psi^{\beta}-\psi^{* \beta} \psi^{\alpha}\right)\right] .
\end{aligned}
$$

This model is connected with chiral field on the orthogonal group $O(N)$. The method developed here makes it possible to find explicit soliton solutions of the classical spinor systems under investigation.

\section{Some Information from the Inverse Scattering Method}

1. The integrable systems under investigation are the compatibility conditions for the system of two linear equations [5]:

$$
\begin{aligned}
& \Psi_{\xi}=U \Psi \\
& \Psi_{\eta}=V \Psi .
\end{aligned}
$$

Here $\eta=t+x, \xi=t-x$ are light cone variables, and $U, V$ are rational functions of the parameter $\lambda$ :

$$
\begin{gathered}
U=U_{0}+\sum_{n=1}^{K} \frac{U_{n}}{\lambda-a_{n}} \\
V=V_{0}+\sum_{n=1}^{K} \frac{V_{n}}{\lambda+a_{n}},
\end{gathered}
$$

where $a_{n}$ are arbitrary complex constants, here $a_{n}+a_{m} \neq 0 ; U, V, \Psi$ are complex $N \times N$ matrices. The system (1.1) should be compatible for any $\lambda$.

The compatibility conditions have the form:

$$
\begin{aligned}
& U_{0 \eta}-V_{0 \xi}+\left[U_{0} V_{0}\right]=0 \\
& U_{n \eta}+\left[U_{n}, \Phi_{n}\right]=0, \\
& V_{n \xi}+\left[V_{n}, \Psi_{n}\right]=0, \\
& \Phi_{n}=V_{0}+\sum_{m=1}^{K} \frac{V_{m}}{a_{n}+a_{m}} \\
& \Psi_{n}=U_{0}-\sum_{m=1}^{K} \frac{U_{m}}{a_{n}+a_{m}} .
\end{aligned}
$$


2. Consider the change of variables

$$
X=g \Psi
$$

in the problem (1.1) where $g(\xi, \eta, \lambda)$ is some nondegenerate matrix function. With such a substitution, Eqs. (1.1) are transformed to the form:

$$
\begin{aligned}
& \partial_{\xi} X=\tilde{U} X \\
& \partial_{\eta} X=\tilde{V} X,
\end{aligned}
$$

where

$$
\begin{aligned}
& \tilde{U}=g_{\xi} g^{-1}+g U g^{-1} \\
& \tilde{V}=g_{\eta} g^{-1}+g V g^{-1} .
\end{aligned}
$$

If $g$ is independent of $\lambda$, then $\tilde{U}$ and $\tilde{V}$ are rational functions with poles at the same points as $U$ and $V$. Therefore, the compatibility conditions of the problem (1.7) have the form of (1.3)-(1.5) where instead of $U_{0}, V_{0}, U_{n}, V_{n}$ the transformed matrices $\tilde{U}_{0}, \tilde{V}_{0}$ and $\tilde{U}_{n}, \tilde{V}_{n}$ appear:

$$
\begin{aligned}
& \tilde{U}_{0}=g U_{0} g^{-1}+g_{\xi} g^{-1}, \quad \tilde{U}_{n}=g U_{n} g^{-1}, \\
& \tilde{V}_{0}=g V_{0} g^{-1}+g_{\eta} g^{-1}, \quad \tilde{V}_{n}=g V_{n} g^{-1} .
\end{aligned}
$$

Transformations of the form (1.6) have been introduced in $[5,6]$ and are called gauge transformations.

Different choices of the matrix $g$ correspond to different gauges. For instance, the gauge for which $\tilde{U}_{0}=\tilde{V}_{0}=0$ (the matrix $g=g_{0}$ is determined from the equations $g_{0 \xi}+U_{0} g_{0}=0, g_{0 \eta}+V_{0} g_{0}=0$ up to multiplication by a constant matrix) is called canonical; the gauge in which one of the residue matrices, for instance $\tilde{V}_{K}$, is of Jordan canonical form, is called a pole gauge $\left(g=g_{K}\right.$ is determined as a matrix reducing $V_{K}$ to Jordan form) [5].

Equations (1.3), (1.4) in different gauges have at first sight different forms; however, it is clear that their solutions are connected, and it is sufficient to study them in a single gauge.

3. It follows from (1.4) that the invariants of the matrices are independent of $\eta$. Thus

$$
U_{n}=\varphi_{n} U_{n}^{0}(\xi) \varphi_{n}^{-1} .
$$

Similarly:

$$
V_{n}=\psi_{n} V_{n}^{0}(\xi) \psi_{n}^{-1} \text {. }
$$

In other words the invariants of the residue matrices are first integrals of the system (1.3), (1.4) which actually falls into a set of systems differing in the predetermination of the values of the invariants. Systems with different sets of invariants may result in solutions which differ qualitatively (see $[5,7]$ ), and, therefore, each of these systems should be studied separately.

It should be noted that the gauge transformations do not affect the values of the invariants $U_{n}$ and $V_{n}$.

4. To construct exact solutions of system (1.3), (1.4) by the inverse scattering method it is required that at least one particular solution of this system be known. 
It would be natural to start from a solution which is homogeneous in space-time, "from the classical vacuum", on which background the dynamics would develop.

A set of matrices $V_{0}^{0}(\eta), U_{0}^{0}(\xi), V_{n}^{0}(\eta), U_{n}^{0}(\xi)$ satisfying the following system of algebraic equations will be called a vacuum solution:

$$
\begin{aligned}
& {\left[U_{0}^{0}, V_{0}^{0}\right]=0, \quad\left[U_{n}^{0}, \Phi_{n}^{0}\right]=0, \quad\left[V_{n}^{0}, \Psi_{n}^{0}\right]=0,} \\
& \Phi_{n}^{0}=V_{0}^{0}+\sum_{m=1}^{K} \frac{V_{m}^{0}}{a_{n}+a_{m}}, \quad \Psi_{n}^{0}=U_{0}^{0}-\sum_{m=1}^{K} \frac{U_{m}^{0}}{a_{n}+a_{m}},
\end{aligned}
$$

when the gauge of problem (1.13), (1.14) coincides with that of system (1.3), (1.4), this set of matrices will be called a first order vacuum solution of system (1.3), (1.4). When the gauges are different then, after the corresponding gauge transformation reducing problem (1.13), (1.14) to the same gauge as (1.3), (1.4), we get a set of matrices $\tilde{U}_{0}^{0}(\xi, \eta), \tilde{V}_{0}^{0}(\xi, \eta), \tilde{U}_{n}^{0}(\xi, \eta), \tilde{V}_{n}^{0}(\xi, \eta)$ which is the solution of system (1.3), (1.4), which we call a second order vacuum solution of system (1.3), (1.4).

Almost all the systems considered earlier have been considered on the first order vacuum background. An example of studying a problem on the second order vacuum background solution can be found in the paper of Shabat and one of the authors [8]. Below we show that for the Gross-Neveu model only the second order vacuum leads to a nontrivial dynamics.

In this paper we shall mainly deal with systems given in the canonical gauge $U_{0}$ $=V_{0}=0$. To diminish the number of indices we restrict ourselves to the problem with one pole $a_{1}=1$. In this case the system (1.3), (1.4) takes the form

$$
\begin{aligned}
& \partial_{\eta} U_{1}=1 / 2\left[V_{1} U_{1}\right], \\
& \partial_{\xi} V_{1}=1 / 2\left[V_{1} U_{1}\right] .
\end{aligned}
$$

The compatibility conditions of (1.13), (1.14) for the first order vacuum solution have the form

$$
\left[U_{1}^{0}(\xi), V_{1}^{0}(\eta)\right]=0,
$$

where the matrices $U_{1}^{0}(\xi), V_{1}^{0}(\eta)$ satisfy system (1.15), (1.16). For the second order vacuum the compatibility conditions are as follows:

$$
\begin{aligned}
& {\left[U_{0}^{0}(\xi), V_{0}^{0}(\eta)\right]=0,} \\
& {\left[U_{1}^{0}(\xi), V_{0}^{0}(\eta)+\frac{1}{2} V_{1}^{0}(\eta)\right]=0,} \\
& {\left[V_{1}^{0}(\eta) ; U_{0}^{0}(\xi)-\frac{1}{2} U_{1}^{0}(\xi)\right]=0 .}
\end{aligned}
$$

In order to obtain the second order vacuum solution of (1.15), (1.16) one should perform a gauge transformation [Eqs. (1.18), (1.19) must be transformed to the canonical gauge]. It follows from the foregoing that it is necessary to find the matrix $g^{0}(\xi, \eta)$ satisfying the consistent system of equations

$$
\partial_{\xi} g^{0}+U_{0}^{0} g^{0}=0, \quad \partial_{\eta} g^{0}+V_{0}^{0} g^{0}=0
$$

1 It goes without saying that this restriction is not essential and that all results can be easily generalized to the $K$-pole problem 
and then using it to perform the gauge transformation of $(1.8)$ :

$$
\begin{aligned}
& \tilde{U}_{1}^{0}=g^{0} U_{1}^{0} g^{0-1} \\
& \tilde{V}_{1}^{0}=g^{0} V_{1}^{0} g^{0-1}
\end{aligned}
$$

(leading to $\left.\tilde{U}_{0}^{0}=\tilde{V}_{0}^{0}=0\right)$. The matrices $\tilde{U}_{1}^{0}(\xi, \eta)$ and $V_{1}^{0}(\xi, \eta)$ satisfy system $(1.15)$, (1.16). They are called the second order vacuum solution.

It should be noted that the vacuum solution matrices each depend only on a single variable, in particular, they may be constant, and the vacuum solution of system (1.15), (1.16) of the second order (1.21) must necessarily depend on both coordinates.

5. Let us consider Eqs. (1.1), where $U, V$ are the vacuum solutions $\left\{U_{0}^{0}(\xi), V_{0}^{0}(\eta)\right.$, $\left.U_{1}^{0}(\xi), V_{1}^{0}(\eta)\right\}$ of Eqs. (1.13), (1.14). The matrix $\Psi^{0}$, which solves

$$
\begin{aligned}
& \partial_{\xi} \Psi^{0}=U^{0}(\xi, \lambda) \Psi^{0}, \\
& \partial_{\eta} \Psi^{0}=V^{0}(\eta, \lambda) \Psi^{0}
\end{aligned}
$$

can be sought in the form of a product of functions

$$
\Psi^{0}(\xi, \eta, \lambda)=\Psi_{1}^{0}(\xi, \lambda) \Psi_{2}^{0}(\eta, \lambda),
$$

where $\Psi_{1}^{0}(\xi, \lambda)$ commutes with $\Psi_{2}^{0}(\eta, \lambda)$.

If the matrices $U_{0}^{0}, V_{0}^{0}, U_{n}^{0}, V_{n}^{0}$ are constant (the most interesting case), then Eqs. (1.22) and (1.23) can be easily solved. Below we assume the functions $\Psi_{1,2}^{0}$ to be known.

It should be noted that the function $\Psi^{0}$ provides complete information about the vacuum.

The matrix $X$ is : $X=\Psi \Psi^{0-1}$. It apparently satisfies the equations

$$
\begin{aligned}
& \partial_{\xi} X=U X-X U^{0} \\
& \partial_{\eta} X=V X-X V^{0} .
\end{aligned}
$$

Let us denote $g=\left.X\right|_{\lambda=\infty}$. Then $\lambda \rightarrow \infty$ in (1.25) we get

$$
U_{0}=g_{\xi} g^{-1}+g U_{0}^{0} g^{-1}, \quad V_{0}=g_{\eta} g^{-1}+g V_{0}^{0} g^{-1} \text {. }
$$

Assuming $\lambda \rightarrow a_{n}$ in (1.25) we yield

$$
\begin{aligned}
& U_{n}=X_{n} U_{n}^{0} X_{n}^{-1}, \quad X_{n}=\left.X\right|_{\lambda=a_{n}}, \\
& \partial_{\eta} X_{n}=\Phi_{n} X_{n}-X_{n} \Phi_{n}^{0} .
\end{aligned}
$$

Analogously $\lambda \rightarrow-a_{n}$ leads to

$$
\begin{aligned}
& \partial_{\xi} \tilde{X}_{n}=\Psi_{n} \tilde{X}_{n}-\tilde{X}_{n} \Psi_{n}^{0}, \\
& V_{n}=\tilde{X}_{n} V_{n}^{0} \tilde{X}_{n}^{-1}, \quad \tilde{X}_{n}=\left.X\right|_{\lambda=-a_{n}} .
\end{aligned}
$$

Below we shall construct a number of explicit expressions for $X$ and thus find exact solutions of Eqs. (1.3), (1.4), and (1.27)-(1.30).

6. Equations (1.3), (1.4) contain only linear operations and commutators. We may assume therefore that the matrices $U$ and $V$ belong to a Lie algebra $\mathfrak{g}$. It is 
clear that any subalgebra $\mathfrak{g}^{\prime} \subset \mathfrak{g}$ is an invariant of system (1.3), (1.4). In other words, if the initial conditions are given in some subalgebra, then in the process of evolution the fields stay in the same subalgebra.

The problem of restricting to invariant manifolds in integrable systems is called the problem of reduction. In this sense the restriction of system (1.3), (1.4) to the subalgebra is reduction.

Below we shall assume all poles to be real. The algebra $\mathfrak{g}$ is $\mathfrak{g}=\operatorname{sl}(N, \mathbb{C})$, and from (1.1) it follows that $\Psi(\xi, \eta, \lambda)$ belongs to the group $\operatorname{SL}(N, \mathbb{C})$ of most importance for us is the reduction to:

i) $\mathfrak{g}^{\prime}=\mathrm{SU}(N)$ - the algebra of anti-hermitian matrices,

ii) $\mathfrak{g}^{\prime}=\mathrm{Sp}(2 N, \mathbb{R})-$ the algebra of the real symplectic group,

iii) $\mathfrak{g}^{\prime}=\mathrm{SO}(N)$ - the algebra of anti-symmetric matrices.

In the first case of real $\lambda$ the function $\Psi(\xi, \eta, \lambda)$ can be chosen to belong to $U(N)$, in the second case to $\operatorname{Sp}(2 N, \mathbb{R})$, and in the third case to $O(N)$.

We shall mainly consider the simplest case of a single-pole problem in the canonical gauge:

$$
\begin{aligned}
& \partial_{\xi} \Psi=\frac{U_{1}}{\lambda-1} \Psi, \\
& \partial_{\eta} \Psi=\frac{V_{1}}{\lambda+1} \Psi .
\end{aligned}
$$

From (1.31), (1.32) it follows that the matrix $g=\left.\Psi(\xi, \eta, \lambda)\right|_{\lambda=0}$ satisfies the equations

$$
g_{\xi \eta}=\frac{1}{2}\left(g_{\xi} g^{-1} g_{\eta}+g_{\eta} g^{-1} g_{\xi}\right)
$$

which are the field equations describing the principal chiral field on the group (for instance, on one of the groups enumerated above) [5].

It goes without saying that each reduction imposes certain limitations on these scattering problems (or the Riemann problems). Reduction on $\mathrm{SU}(N)$ has been studied in [5]. Reductions on the real algebras $\operatorname{Sp}(2 N, \mathbb{R})$ and $\operatorname{SO}(N)$ are studied in Sect. 3. A detail analysis of these reductions will be published elsewhere.

\section{Correspondence of the Classical Spinor Systems to the Chiral Field Models}

Chiral fields on Lie groups have been investigated in [5]. They may be regarded as representatives of equivalence classes of integrable two-dimensional relativisticinvariant systems. A classical spinor system is shown to be connected in a natural way with each such system.

Let us transform Eqs. (1.28), (1.29) to relativistically invariant form. To this purpose we determine the function $\varphi_{n}$ and $\psi_{n}$ as follows

$$
\begin{aligned}
& \varphi_{n}=X_{n}(\xi, \eta, \lambda) \mathrm{g}^{0}(\xi, \eta) \Psi_{2}^{0}\left(\eta, a_{n}\right), \\
& \psi_{n}=\tilde{X}_{n}(\xi, \eta, \lambda) g^{0}(\xi, \eta) \Psi_{1}^{0}\left(\xi,-a_{n}\right) .
\end{aligned}
$$

[Here $X_{n}, \tilde{X}_{n}, g^{0}, \Psi_{1,2}^{0}$ are determined in formulas (1.27), (1.30), (1.20), and (1.24).] They evidently satisfy the system

$$
\varphi_{n \eta}=\Phi_{n} \varphi_{n}, \quad \psi_{n \xi}=\Psi_{n} \psi_{n}
$$


which can be rewritten in the form

$$
\begin{aligned}
& \nabla_{n} \varphi_{n}=\sum_{m=1}^{K} \frac{\psi_{m} V_{m}^{0} \psi_{m}^{-1}}{a_{n}+a_{m}} \varphi_{n}, \\
& \nabla_{\xi} \psi_{n}=-\sum_{m=1}^{K} \frac{\varphi_{m} U_{m}^{0} \varphi_{m}^{-1}}{a_{n}+a_{m}} \psi_{n},
\end{aligned}
$$

where $\nabla_{\eta}=\partial_{\eta}-V_{0}, \nabla_{\xi}=\partial_{\xi}-U_{0},\left[\nabla_{\eta}, \nabla_{\xi}\right]=0$.

Equations (2.3), (2.4) are of the form of a classical spinor field $\left(\varphi_{n}, \psi_{n}\right.$ form a two-component Dirac spinor) and are obviously relativistic-invariant.

Equations (2.3), (2.4) possess a variational principle [9]. Let us consider the functional

$$
\begin{aligned}
S= & \int d \eta d \xi S_{p}\left[-\sum_{n=1}^{K}\left(\varphi_{n}^{-1} \nabla_{\eta} \varphi_{n} U_{n}^{0}-\psi_{n}^{-1} \nabla_{\xi} \psi_{n} V_{n}^{0}\right)\right. \\
& \left.+\sum_{n, m}^{K}\left(a_{n}+a_{m}\right)^{-1} \varphi_{n} U_{n}^{0} \varphi_{n}^{-1} \psi_{m} V_{m}^{0} \psi_{m}^{-1}\right]
\end{aligned}
$$

and calculate its variation taking account of the fact that

$$
\delta \psi_{n}^{-1}=-\psi_{n}^{-1} \delta \psi_{n} \psi_{n}^{-1}, \quad \delta \varphi_{n}^{-1}=-\varphi_{n}^{-1} \delta \varphi_{n} \varphi_{n}^{-1}
$$

The condition $\delta S=0$ is equivalent to the following equations (variation with respect to $\left.\varphi_{n}, \psi_{n}\right)$ :

$$
\begin{aligned}
& \left(\nabla_{\eta}-\sum_{m=1}^{K} \frac{\psi_{m} V_{m}^{0} \psi_{m}^{-1}}{a_{n}+a_{m}}\right) \varphi_{n} U_{n}^{0}=U_{n}\left(\nabla_{\eta}-\sum_{m=1}^{K} \frac{\psi_{m} V_{m}^{0} \psi_{m}^{-1}}{a_{n}+a_{m}}\right) \varphi_{n}, \\
& \left(\nabla_{\xi}+\sum_{m=1}^{K} \frac{\varphi_{m} U_{m}^{0} \varphi_{m}^{-1}}{a_{n}+a_{m}}\right) \psi_{n} V_{n}^{0}=V_{n}\left(\nabla_{\xi}+\sum_{m=1}^{K} \frac{\varphi_{m} U_{m}^{0} \varphi_{m}^{-1}}{a_{n}+a_{m}}\right) \psi_{n},
\end{aligned}
$$

and to the equation (variation in $g$ )

$$
\left[\nabla_{\eta}, \sum_{n=1}^{K} U_{n}\right]=\left[\nabla_{\xi}, \sum_{n=1}^{K} V_{n}\right] \text {. }
$$

Equation (2.2) can be solved, using (1.27), in the following way:

$$
\left(\nabla_{\eta}-\sum_{m=1}^{K} \frac{\psi_{m} V_{m}^{0} \psi_{m}^{-1}}{a_{n}+a_{m}}\right) \varphi_{n}=\varphi_{n} Q_{n},
$$

where $Q_{n}(\xi, \eta)$ is any matrix commutating with $U_{n}^{0}(\xi)$. In particular, $\delta S=0$ is satisfied provided $Q_{n}=0$.

It may seem that the functional does not unambiguously determine the equation for the matrix $\varphi_{n}$. All the ambiguity is, however, connected with multiplication of the matrix $\varphi_{n}$ on the right by an arbitrary matrix commutating with $U_{n}^{0}(\xi)$. Indeed, from $\left[Q_{n}(\xi, \eta), U_{n}^{0}(\xi, \eta)\right]$ it follows that it is possible to find a matrix $R_{n}(\xi, \eta)$ satisfying the equation

$$
R_{n \eta}=R_{n} Q_{n}
$$


and commuting with $U_{n}^{0}(\xi)$. It is easy to verify that the transformation $\varphi_{n} \rightarrow \varphi_{n} R_{n}$ does not change the functional $S$; in this case Eq. (2.9) transforms to (2.3), (2.4). This fact shows that any representative of the "flag space" connected with the matrix $U_{n}^{0}(\xi)$ can be chosen as $\varphi_{n}$ (see [5]). So, without loss of generality, we may assume that the functional $S$ unambiguously determines Eqs. (2.3), (2.4). It can easily be verified that Eq. (2.8) is their consequence.

Equations (2.3), (2.4) are gauge-invariant in the sense that they do not impose any conditions upon the matrix $g$. Therefore, the transformations $\varphi_{n} \rightarrow h \varphi_{n}$, $\psi_{n} \rightarrow h \psi_{n}$ are admissible, where $h$ is an arbitrary matrix depending on $\xi$ and $\eta$. Under such a transformation only the matrix $g$ will be changed. It is possible to get the final determination of system (2.3), (2.4) by imposing an additional condition specifying unambiguously the matrix $g$. Undoubtedly, it is the variational principle for all such systems. In this sense the matrices $\varphi_{n}, \psi_{n}$ should be regarded as variable ones, in which the variational principle is formulated in a most simple manner.

In view of the recent paper [10] it seems quite probable that the variational principle predetermined by the functional (2.5) is not the only variational principle determining Eqs. (2.3), (2.4). At any rate, this is the case for the sine-Gordon equation. We may show that the functional $S$ calculated for the case when system (2.3), (2.4) is equivalent to this equation (see [5]), differs from the natural action functional for the sine-Gordon equation.

\section{Construction of Soliton Solutions}

1. The study of analyticity properties of the function $X(1.24)$ for complex values of the parameter $\lambda$ is very important for constructing exact solutions by the inverse method. The solution of system (1.3), (1.4) corresponding to the case when the function $X$ is rational we shall call a soliton solution. In [11] it has been shown that the calculation of such solutions is a problem in linear algebra and can be solved explicitly. In $[5,7]$ the simplest soliton solutions have been investigated for the principal chiral field on $\mathrm{SU}(N)$. In the present paper we consider soliton solutions for principal chiral fields on the real matrix groups as well.

The choice of the group [fixed reduction in system (1.3), (1.4)] imposes restrictions on the number and position of the poles of the function $X$. The solution of system (1.3), (1.4), for which $X$ has the minimum number of poles, will be called a single-soliton solution. In this case one should take into consideration that the function $X$ is determined up to multiplication by a constant rational matrix function of $\lambda$ commuting with $U^{0}, V^{0}$.

2. We start with the case of the chiral field on the $\mathrm{SU}(N)$ group. From (1.25) it follows that $U, V$ belong to the algebra of antihermitian matrices, provided the function $X$ obeys the involution

$$
X(\lambda) X^{\dagger}(\bar{\lambda})=I
$$

and for real values of the parameter $\lambda$ is unitary. Involution (3.1) does not impose any restrictions upon the arrangement of poles. Multiplying by some appropriate rational function it is possible, as is shown in [5], to transfer all the poles into a fixed (upper or lower) half-plane. In this case the single-solution considered in $[5,7]$ corresponds to the function $X$, having only one pole. However, bearing in 
mind a further comparison with the real case, we shall assume the poles of $X$ to be located at complex-conjugate points. Since the gauge is canonical, it can be presented as follows:

$$
X=\overline{1}+\sum_{n}\left(\frac{A_{n}}{\lambda-\lambda_{n}}+\frac{B_{n}}{\lambda-\bar{\lambda}_{n}}\right) .
$$

From (3.1) it follows that

$$
X^{-1}=1+\sum_{n}\left(\frac{A_{n}^{\dagger}}{\lambda-\bar{\lambda}_{n}}+\frac{B_{n}^{\dagger}}{\lambda-\lambda_{n}}\right) .
$$

From $X^{-1} X=X X^{-1}=I$ we find

$$
\begin{aligned}
& A_{n} B_{n}^{\dagger}=B_{n} A_{n}^{\dagger}=0 \\
& A_{n} \omega_{n}^{\dagger}+\tau_{n} B_{n}^{\dagger}=0 \\
& \omega_{n}=I+\sum_{m} \frac{A_{m}}{\lambda_{n}-\bar{\lambda}_{m}}+\sum_{m \neq n} \frac{B_{m}}{\lambda_{n}-\lambda_{m}} \\
& \tau_{n}=I+\sum_{m \neq n} \frac{A_{m}}{\lambda_{n}-\lambda_{m}}+\sum_{m} \frac{B_{m}}{\lambda_{n}-\bar{\lambda}_{m}} .
\end{aligned}
$$

From (3.3) it follows that the matrices $A_{n}$ and $B_{n}$ are degenerated.

The system of quadratic relations (3.4) can be solved as follows. Let us write $A_{n}$ and $B_{n}$ in the factorized form

$$
A_{n}=X_{n} F_{n}^{\dagger}, \quad B_{n}=Y_{n} G_{n}^{\dagger},
$$

where $X_{n}, F_{n}$ are rectangular matrices made up of $N$ lines and $K_{n}$ columns; $Y_{n}, G_{n}$ have $N$ lines and $S_{n}$ columns. The matrices $F_{n}$ and $G_{n}$ are known, and $X_{n}$ and $Y_{n}$ are unknown. From (3.4) it follows that the following condition should be satisfied:

$$
F_{n}^{\dagger} G_{n}=0
$$

and also the relations

$$
X_{n}\left(F_{n}^{\dagger} \omega_{n}^{\dagger}\right)+\left(\tau_{n} G_{n}\right) Y_{n}^{\dagger}=0 .
$$

Expression (3.7) can be identically rewritten in the form

$$
\tau_{n} G_{n}=-X_{n} \alpha_{n}, \quad \omega_{n} F_{n}=Y_{n} \alpha_{n}^{\dagger},
$$

where $\alpha_{n}$ is some $K_{n} \times S_{n}$ matrix. Relations (3.8) represent a system of linear equations for $X_{n}, Y_{n}$ :

$$
\begin{aligned}
& G_{n}+\sum_{m \neq n} \frac{X_{m}\left(F_{m}^{\dagger} G_{n}\right)}{\lambda_{n}-\lambda_{m}}+\sum_{m} \frac{Y_{m}\left(G_{m}^{\dagger} G_{n}\right)}{\lambda_{n}-\bar{\lambda}_{m}}=-X_{n} \alpha_{n}, \\
& F_{n}+\sum_{m} \frac{X_{m}\left(F_{m}^{\dagger} F_{m}\right)}{\lambda_{n}-\bar{\lambda}_{m}}+\sum_{m \neq n} \frac{Y_{m}\left(G_{m}^{\dagger} F_{n}\right)}{\lambda_{n}-\lambda_{m}}=Y_{n} \alpha_{n}^{\dagger} .
\end{aligned}
$$

3. From formula (1.25) it follows that

$$
U=-X\left(\partial_{\xi}-U^{0}\right) X^{-1} \text {. }
$$


We substitute $X$ into (3.11) in the form of (3.2). In order that $U$ be a solution of (1.3)-(1.5) it is necessary and sufficient that $U$ should represent a rational function with poles at the points $a_{n}$ (1.2). We assume that the points $\lambda_{n}$ and $\bar{\lambda}_{n}$ coincide with none of the $a_{n}$. Therefore, the function $U$ at the points $\lambda_{n}, \bar{\lambda}_{n}$ should be regular, though formally it may have at these points poles of up to second order. The requirement of the absence of second order poles leads to the equations:

$$
\begin{aligned}
& F_{n}^{\dagger} D\left(\lambda_{n}\right) G_{n}=0 \\
& G_{n}^{\dagger} D\left(\bar{\lambda}_{n}\right) F_{n}=0,
\end{aligned}
$$

where

$$
D\left(\lambda_{n}\right)=\partial_{\xi}-\left.U^{0}\right|_{\lambda=\lambda_{n}}
$$

which due to (3.6) can be solved in the following way

$$
\begin{aligned}
& D\left(\lambda_{n}\right) G_{n}=G_{n} \beta_{n} \\
& D\left(\bar{\lambda}_{n}\right) F_{n}=F_{n} \gamma_{n} .
\end{aligned}
$$

Analogously

$$
\begin{aligned}
& \tilde{D}\left(\lambda_{n}\right) G_{n}=G_{n} \tilde{\beta}_{n} \\
& \tilde{D}\left(\bar{\lambda}_{n}\right) F_{n}=F_{n} \tilde{\gamma}_{n},
\end{aligned}
$$

where

$$
\tilde{D}\left(\lambda_{n}\right)=\partial_{\eta}-\left.V^{0}\right|_{\lambda=\lambda_{n}}
$$

$\beta_{n}, \tilde{\beta}_{n}, \gamma_{n}, \tilde{\gamma}_{n}$ are some matrices that, generally speaking, depend on $\xi$ and $\eta$.

From the compatibility condition of (3.13), (3.14) we have

$$
\begin{aligned}
& \frac{\partial \beta_{n}}{\partial \eta}-\frac{\partial \tilde{\beta}_{n}}{\partial \xi}+\left[\beta_{n} \tilde{\beta}_{n}\right]=0 \\
& \frac{\partial \gamma_{n}}{\partial \eta}-\frac{\partial \tilde{\gamma}_{n}}{\partial \xi}+\left[\gamma_{n} \tilde{\gamma}_{n}\right]=0 .
\end{aligned}
$$

Then

$$
\begin{array}{ll}
\beta_{n}=g_{n \xi} g_{n}^{-1}, & \tilde{\beta}_{n}=g_{n \eta} g_{n}^{-1}, \\
\gamma_{n}=h_{n \xi} h_{n}^{-1}, & \tilde{\gamma}_{n}=h_{n \eta} h_{n}^{-1},
\end{array}
$$

where $g, h$ are any non-degenerate $S_{n} \times S_{n}$ and $K_{n} \times K_{n}$ matrices.

The factorization (3.5) is ambiguous. Indeed, if the transformation

$$
F_{n} \rightarrow F_{n} f_{n}^{\dagger}, \quad X_{n} \rightarrow X_{n} f_{n}^{-1}
$$

is performed with an arbitrary non-degenerate matrix $f_{n}$, then $A_{n}$ is not changed. The freedom in the choice of the matrices $f_{n}$ is just connected with some freedom in factorization, and an arbitrary choice of these matrices does not change the final result. We choose these matrices in the simplest way, requiring that

$$
\beta_{n}=\tilde{\beta}_{n}=\gamma_{n}=\tilde{\gamma}_{n}=0 \text {. }
$$


Now the solution of Eqs. (3.13), (3.14) has the form

$$
\begin{aligned}
& G_{n}(\xi, \eta)=\Psi^{0}\left(\xi, \eta, \lambda_{n}\right) G_{n}^{0} \\
& F_{n}(\xi, \eta)=\Psi^{0}\left(\xi, \eta, \bar{\lambda}_{n}\right) F_{n}^{0},
\end{aligned}
$$

where $F_{n}^{0}, G_{n}^{0}$ are constant matrices satisfying the condition (3.6), and $\Psi^{0}(\xi, \eta, \lambda)$ is one of the solutions of system (1.1) satisfying the condition

$$
\Psi^{0}(\xi, \eta, \lambda) \Psi^{0 \dagger}(\xi, \eta, \bar{\lambda})=I \text {. }
$$

The last condition is necessary for the requirement (3.6) to be satisfied at any point of the $\lambda$-plane. As a rule, a vacuum "wave function" of the first or of the second order is chosen as $\Psi^{0}$.

It is also necessary to require that there be no first order poles in expression (3.11). Imposing zero residue at the point $\lambda_{n}=\lambda_{n}$ results in the equation

$$
A_{n} D\left(\lambda_{n}\right) \omega_{n}^{\dagger}+\tau_{n} D\left(\lambda_{n}\right) B_{n}^{\dagger}=\left.A_{n} \frac{\partial U^{0}}{\partial \lambda}\right|_{\lambda=\lambda_{n}} B_{n}^{\dagger} .
$$

Taking account of (3.5), (3.17) we reduce Eq. (3.18) to the following:

$$
X_{n} F_{n}^{\dagger} D\left(\lambda_{n}\right) \omega_{n}^{\dagger}-X_{n} \alpha_{n} \partial_{\xi} Y_{n}^{\dagger}=\left.X_{n} F_{n}^{\dagger} \frac{\partial U^{0}}{\partial \lambda}\right|_{\lambda=\lambda_{n}} G_{n} Y_{n}^{\dagger} .
$$

Differentiating relation (3.8) with respect to $\xi$

$$
\left(\partial_{\xi} F_{n}^{\dagger}\right) \omega_{n}^{\dagger}+F_{n}^{\dagger} \partial_{\xi} \omega_{n}^{\dagger}=\left(\partial_{\xi} \alpha_{n}\right) Y_{n}^{\dagger}+\alpha_{n} \partial_{\xi} Y_{n}^{\dagger}
$$

and using the equation for $F$ :

$$
\begin{aligned}
& \partial_{\xi} F_{n}^{\dagger}+\left.F_{n}^{\dagger} U^{0}\right|_{\lambda=\lambda_{n}}=0 \\
& U^{0 \dagger}\left(\bar{\lambda}_{n}\right)=-U^{0}\left(\lambda_{n}\right)
\end{aligned}
$$

we obtain

$$
F_{n}^{\dagger}\left(\partial_{\xi}-U^{0}\right) \omega_{n}^{\dagger}=\left(\partial_{\xi} \alpha_{n}\right) Y_{n}^{\dagger}+\alpha_{n} \partial_{\xi} Y_{n}^{\dagger} .
$$

Inserting (3.20) into (3.19) we get

$$
X_{n}\left(\partial_{\zeta} \alpha_{n}\right) Y_{n}^{\dagger}=\left.X_{n} F_{n}^{\dagger} \frac{\partial U^{0}}{\partial \lambda}\right|_{\lambda=\lambda_{n}} G_{n} Y_{n}^{\dagger \operatorname{def}} X_{n} \alpha_{n}^{1} Y_{n}^{\dagger}
$$

In a similar way we have

$$
X_{n}\left(\partial_{\eta} \alpha_{n}\right) Y_{n}^{\dagger}=\left.X_{n} F_{n}^{\dagger} \frac{\partial V^{0}}{\partial \lambda}\right|_{\lambda=\lambda_{n}} G_{n} Y_{n}^{\dagger \operatorname{def}} X_{n} \alpha_{n}^{2} Y_{n}^{\dagger} .
$$

One can verify that $\alpha_{n}^{\prime}=\alpha_{n}^{\prime}(\xi)$ and $\alpha_{n}^{2}=\alpha_{n}^{2}(\eta)$. Consequently, in order to cancel the first order poles it is necessary and sufficient to put $\alpha_{n}=S^{\xi} d \xi^{\prime} d_{n}^{1}+S^{\eta} d \eta^{\prime} \alpha_{n}^{2}$ and $\alpha_{n}^{0}$ is an arbitrary constant.

The scheme of calculation of the function $X$ for the soliton solutions can be formulated as follows. Each $N$ soliton (i.e., the one corresponding to $2 N$ poles of the function $X$ ) solution is given by: a set of $N$ rectangular constants $N \times S_{n}$ matrices $G_{n}^{0}$, a set of $N$ rectangular constants $K_{n} \times N$ of the matrices $F_{n}^{0}$, a set of rectangular constants $K_{n} \times S_{n}$ of the matrices $\alpha_{n}^{0}$, a set of $N$ complex numbers $\lambda_{n}$, and the matrices $F_{n}^{0}$ and $G_{n}^{0}$ satisfying the condition (3.6). The matrices $G_{n}(\xi, \eta)$ and $F_{n}(\xi, \eta)$ are calculated by formulas (3.17), and the matrices $X_{n}, Y_{n}$ are determined 
from the solution of Eqs. (3.9), (3.10). Then constructing $A_{n}$ and $B_{n}(3.5)$ we find the function $X$ (3.2) corresponding to the $N$ soliton solution.

We consider now the case of a unitary group. In order to turn to the orthogonal group it is sufficient to require that the function $X$ should be real at real $\lambda$. In this case $A_{n}=\bar{B}_{n}$, that is,

$$
G_{n}=\bar{F}_{n}, \quad Y_{n}=\bar{X}_{n}, \quad K_{n}=S_{n}, \quad \alpha_{n}=-\alpha_{n}^{\text {tr }} .
$$

4. In a similar way it is possible to consider any classical real matrix group. Such groups are characterized by the invariant form $\mathscr{J}$

$$
g^{\text {tr }} \mathscr{J} g=\mathscr{J}
$$

which can be reduced to any of the two canonical types

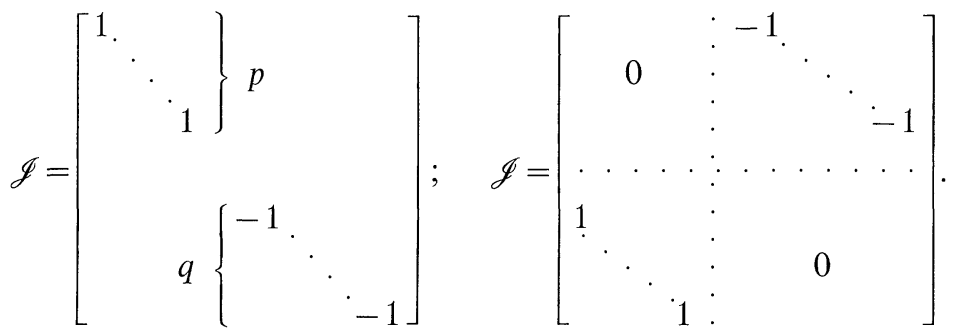

The group preserving the form (3.22) is called the pseudoorthogonal $O(p, q)$, and the group preserving (3.23) is called the symplectic group $\operatorname{Sp}(2 N, \mathbb{R})$.

Using the reality of $X$ at real $\lambda$, we have

$$
\begin{aligned}
X & =I+\sum_{n}\left(\frac{A_{n}}{\lambda-\lambda_{n}}+\frac{\bar{A}_{n}}{\lambda-\bar{\lambda}_{n}}\right), \\
X^{-1} & =\mathscr{J} X^{\mathrm{tr}} \mathscr{J}^{-1} .
\end{aligned}
$$

Assuming

$$
A_{n}=X_{n} F_{n}^{\dagger}
$$

we see that

$$
F_{n}^{\operatorname{tr}} \mathscr{J} F_{n}=0 \text {. }
$$

Proceeding from the above statements we arrive at the equations for $X_{n}$ :

$$
\mathscr{J} \bar{F}_{n}+\sum_{m \neq n} \frac{X_{m}\left(F_{m}^{\dagger} \mathscr{J} \bar{F}_{n}\right)}{\lambda_{n}-\lambda_{m}}+\sum_{m} \frac{\bar{X}_{m}\left(F_{m}^{\operatorname{tr}} \mathscr{J} F_{n}\right)}{\lambda_{n}-\bar{\lambda}_{m}}=-X_{n} \alpha_{n} .
$$

In the case of the symplectic group $\alpha^{\text {tr }}=\alpha$, in the case of the pseudoorthogonal group $\alpha^{\mathrm{tr}}=-\alpha$.

5. To determine the dependence of $F_{n}$ on the coordinates $(\xi, \eta)$ calculations identical to those in Paragraph 3 of this section should be carried out. As a result, we get a generalization of formula (3.16):

$$
\mathscr{J} \bar{F}_{n}=\Psi^{0}\left(\xi, \eta, \lambda_{n}\right) \mathscr{J} \bar{F}_{n}^{0}
$$

(the function $\Psi^{0}$ satisfies the involution $\Psi^{0 \text { tr }} \mathscr{J}=\mathscr{J} \Psi^{0-1}$ ). The matrix $\alpha$, as in Paragraph 3, can be easily computed. 
6. The function $X$ is calculated in the simplest case when there are only two poles at the points $\lambda_{0}$ and $\bar{\lambda}_{0}$. Such a solution for the case of orthogonal (pseudoorthogonal) and symplectic groups is simple and corresponds to a singlesoliton solution.

i) Unitary Group. In the case of a unitary group there is a solution with only one pole $[5,7]$ :

$$
X(\xi, \eta, \lambda)=I-\frac{\lambda_{0}-\bar{\lambda}_{0}}{\lambda-\bar{\lambda}_{0}} F\left(F^{\dagger} F\right)^{-1} F^{\dagger},
$$

where $F(\xi, \eta)=\Psi^{0}\left(\xi, \eta, \lambda_{0}\right) F^{0}, \quad F^{0}$ is an arbitrary constant $N \times K$ matrix $\left(\operatorname{det}\left(F^{0 \dagger} F^{0}\right) \neq 0\right)$. In order to find $X$ with poles at conjugate points the system of matrix Eqs. (3.9), (3.10) should be solved:

$$
\begin{aligned}
& G+Y \frac{G^{\dagger} G}{\lambda_{0}-\bar{\lambda}_{0}}=-X \alpha \\
& F-X \frac{F^{\dagger} F}{\lambda_{0}-\bar{\lambda}_{0}}=Y \alpha^{\dagger} .
\end{aligned}
$$

The answer has the form

$$
\begin{aligned}
X= & {\left[F\left(\lambda_{0}-\bar{\lambda}_{0}\right)-\left|\lambda_{0}-\bar{\lambda}_{0}\right|^{2} G\left(G^{\dagger} G\right)^{-1} \alpha^{\dagger}\right] } \\
& \cdot\left[F^{\dagger} F+\left|\lambda_{0}-\bar{\lambda}_{0}\right|^{2} \alpha\left(G^{\dagger} G\right)^{-1} \alpha^{\dagger}\right]^{-1} \\
Y= & {\left[G\left(\lambda_{0}-\lambda_{0}\right)+\left|\lambda_{0}-\bar{\lambda}_{0}\right|^{2} F\left(F^{\dagger} F\right)^{-1} \alpha\right] } \\
& \cdot\left[G^{\dagger} G+\left|\lambda_{0}-\bar{\lambda}_{0}\right|^{2} \alpha^{\dagger}\left(F^{\dagger} F\right)^{-1} \alpha\right]^{-1} .
\end{aligned}
$$

The final expression for $X$ in this case has the form

$$
\begin{aligned}
X(\xi, \eta, \lambda)= & I+\frac{\lambda_{0}-\bar{\lambda}_{0}}{\lambda-\lambda_{0}}\left[F+\left(\lambda_{0}-\bar{\lambda}_{0}\right) G\left(G^{\dagger} G\right)^{-1} \alpha^{\dagger}\right] \\
& \cdot\left[F^{\dagger} F+\left|\lambda_{0}-\bar{\lambda}_{0}\right|^{2} \alpha\left(G^{\dagger} G\right)^{-1} \alpha^{\dagger}\right]^{-1} F^{\dagger} \\
& -\frac{\lambda_{0}-\bar{\lambda}_{0}}{\lambda-\lambda_{0}}\left[G+\left(\lambda_{0}-\bar{\lambda}_{0}\right) F\left(F^{\dagger} F\right)^{-1} \alpha\right] \\
& \cdot\left[G^{\dagger} G+\left|\lambda_{0}-\bar{\lambda}_{0}\right|^{2} \alpha^{\dagger}\left(F^{\dagger} F\right)^{-1} \alpha\right]^{-1} G,
\end{aligned}
$$

where $F(\xi, \eta)=\Psi^{0}\left(\xi, \eta, \bar{\lambda}_{0}\right) F^{0}, G(\xi, \eta)=\Psi^{0}\left(\xi, \eta, \lambda_{0}\right) G^{0} ; F^{0}$ and $G^{0}$ are arbitrary constant $N \times K_{1}$ and $N \times K_{2}$ matrices; here $F^{0 \dagger} G^{0}=0$ and $\operatorname{det}\left(G^{0 \dagger} G^{0}\right) \neq 0$, $\operatorname{det}\left(F^{0 \dagger} F^{0}\right) \neq 0$.

ii) In the case of real groups Eq. (3.28)

$$
\mathscr{J} \bar{F}+\frac{\bar{X}}{\lambda_{0}-\bar{\lambda}_{0}} F^{\operatorname{tr}} \mathscr{J} \bar{F}=X \alpha
$$

has the solution

$$
\begin{aligned}
X= & \left(\lambda_{0}-\bar{\lambda}_{0}\right)\left[\mathscr{J} F-\left(\lambda_{0}-\bar{\lambda}_{0}\right) \mathscr{J} \bar{F}\left(F^{\operatorname{tr}} \mathscr{J} \bar{F}\right)^{-1} \bar{\alpha}\right] \\
& \cdot\left[F^{\dagger} \mathscr{J} F+\left|\lambda_{0}-\bar{\lambda}_{0}\right|^{2}\left(\alpha^{\dagger}\left(F^{\dagger} \mathscr{J} F\right)^{-1} \alpha\right)^{\mathrm{tr}}\right]^{-1} .
\end{aligned}
$$


Using (3.26), (3.24) we write out the formula for

$$
\begin{aligned}
X(\xi, \eta, \lambda)= & I+\frac{\lambda_{0}-\bar{\lambda}_{0}}{\lambda-\lambda_{0}}\left[\mathscr{J} F-\left(\lambda_{0}-\bar{\lambda}_{0}\right) \mathscr{J} \bar{F}\left(F^{\mathrm{tr}} \mathscr{J} \bar{F}\right)^{-1} \bar{\alpha}\right] \\
& \cdot\left[F^{\dagger} \mathscr{J} F+\left|\lambda_{0}-\bar{\lambda}_{0}\right|^{2}\left(\alpha^{\dagger}\left(F^{\dagger} \mathscr{J} F\right)^{-1} \alpha\right)^{\operatorname{tr}}\right]^{-1} F^{\dagger} \\
& -\frac{\lambda_{0}-\bar{\lambda}_{0}}{\lambda-\bar{\lambda}_{0}}\left[\mathscr{J} \bar{F}+\left(\lambda_{0}-\bar{\lambda}_{0}\right) \mathscr{J} F\left(F^{\dagger} \mathscr{J} F\right)^{-1} \alpha\right] \\
& \cdot\left[F^{\operatorname{tr}} \mathscr{J} F+\left|\lambda_{0}-\bar{\lambda}_{0}\right|^{2} \alpha^{\dagger}\left(F^{\dagger} \mathscr{J} F\right)^{-1} \alpha\right]^{-1} F^{\operatorname{tr}}
\end{aligned}
$$

When $\alpha=0$, formula (3.33) is very much simplified

$$
X=I+\frac{\lambda_{0}-\bar{\lambda}_{0}}{\lambda-\lambda_{0}} \mathscr{J} F\left(F^{\dagger} \mathscr{J} F\right)^{-1} F^{\dagger}-\frac{\lambda_{0}-\bar{\lambda}_{0}}{\lambda-\bar{\lambda}_{0}} \mathscr{J} \bar{F}\left(F^{\mathrm{tr}} \mathscr{J} F\right)^{-1} F^{\mathrm{tr}} .
$$

The matrix $F(\xi, \eta)=\mathscr{J}^{-1} \Psi^{0}\left(\xi, \eta, \lambda_{0}\right) \mathscr{J} F^{0}$, where $F^{0}$ is the constant $N \times K$ matrix, and in this case

$$
F^{0 \operatorname{tr}} \mathscr{J} F^{0}=0, \quad \operatorname{det}\left(F^{\dagger} \mathscr{J} F\right) \neq 0 .
$$

\section{The Nambu, Jona-Lasinio, Vaks, Larkin Model}

1. Let us consider a model of fields on the $U(N)$ group. The compatibility problem for this model has the form (1.31), (1.32), in this case the matrices $U_{1}$ and $V_{1}$ are antihermitian. Suppose the rank of these matrices is unity, then to within a trivial transformation their Jordan form can be given by

$$
V_{1}^{0}=-U_{1}^{0}=i\left(\begin{array}{llll}
1 & & & \\
& 0 & \\
& & \ddots & \\
& & & 0
\end{array}\right) \text {. }
$$

Let us designate the first column of the matrix $\varphi_{1}(2.1)$ as $\varphi^{\alpha}=\left(\varphi_{1}\right)_{\alpha 1}$, the first column of the matrix $\psi_{1}(2.2)$ as $\psi^{\alpha}=\left(\psi_{1}\right)_{\alpha 1}$. It follows from (2.3), (2.4) that they satisfy the equations

$$
\begin{aligned}
& \partial_{\eta} \varphi^{\alpha}=\frac{i}{2} \psi^{\alpha} \sum_{\beta} \psi^{* \beta} \varphi^{\beta} \\
& \partial_{\xi} \psi^{\alpha}=\frac{i}{2} \varphi^{\alpha} \sum_{\beta} \varphi^{* \beta} \psi^{\beta}
\end{aligned}
$$

coinciding with the equations of model (1). The remaining columns $\varphi_{1_{\alpha k}}$ and $\psi_{1_{\alpha k}}$ $(k=2, \ldots N)$ satisfy the system of linear Dirac equations :

$$
\begin{aligned}
& \partial_{\eta} \varphi_{1_{\alpha k}}=\frac{i}{2} \psi^{\alpha} \sum_{\beta} \psi^{* \beta} \varphi_{1_{\beta k}} \\
& \partial_{\xi} \psi_{1_{\alpha k}}=\frac{i}{2} \varphi^{\alpha} \sum_{\beta} \varphi^{* \beta} \psi_{1_{\beta k}}
\end{aligned}
$$


in an external field and impose no restrictions on $\varphi^{\alpha}$ and $\psi^{\alpha}$. Thus, when the functions $X, \Psi_{1,2}^{0}$ are known and satisfy Eqs. (1.31), (1.32), and (1.22), respectively, then it is easy to find the solution of Eqs. (3.2) using formulae (2.1) and (1.2).

2. In order to find the first order vacuum solution of Eqs. (4.2) one should solve the system (1.22), in which $U_{1}^{0} /(\lambda-1)$ and $V_{1}^{0} /(\lambda+1)$ serve as $U^{0}$ and $V^{0}$, respectively. The solution has the form of (1.23), where

$$
\begin{aligned}
& \Psi_{1}^{0}=\exp \left(\frac{U_{1}^{0} \xi}{\lambda-1}\right), \\
& \Psi_{2}^{0}=\exp \left(\frac{V_{1}^{0} \eta}{\lambda+1}\right) .
\end{aligned}
$$

The first order vacuum solution of system (4.2) is determined from formulae (2.1), (2.2), where $X_{n}=\tilde{X}_{n}=I$, and has the form:

$$
\left(\varphi^{\alpha}\right)=\left(\exp \left(\frac{i}{2} \eta\right), 0, \ldots, 0\right) ; \quad\left(\psi^{\alpha}\right)=\left(\exp \left(\frac{i}{2} \xi\right), 0, \ldots, 0\right) .
$$

Let us construct a more general solution of Eqs. (4.2) and show that it corresponds to the second order vacuum. The solution will be sought for as follows :

$$
\begin{aligned}
& \varphi^{\alpha}=A_{\alpha} \exp \left(i \frac{u_{\alpha} \xi}{2}+i \frac{v_{\alpha} \eta}{2}\right) \\
& \psi^{\alpha}=B_{\alpha} \exp \left(i \frac{u_{\alpha} \xi}{2}+i \frac{v_{\alpha} \eta}{2}\right) .
\end{aligned}
$$

Insert (4.6) into (4.2) and determine the relation between the constants:

$$
\begin{aligned}
& v_{\alpha} A_{\alpha}=B_{\alpha} \sum_{\beta} B_{\beta}^{*} A_{\beta} \\
& u_{\alpha} B_{\alpha}=A_{\alpha} \sum_{\beta} A_{\beta}^{*} B_{\beta} .
\end{aligned}
$$

In order for the vacuum solution not to increase in amplitude it is necessary to assume $v_{\alpha}$ and $u_{\alpha}$ to be real. Then it follows from (4.7) that the quantity $\Delta \Theta=\arg A_{\alpha}$ $-\arg B_{\alpha}$ is independent of $\alpha$.

Expressing $u_{\alpha}$ and $v_{\alpha}$ through the sets $\left\{B_{\alpha}\right\}$ and $\left\{A_{\alpha}\right\}$ and inserting them into (4.6) we get a solution more general than (4.5). The matrices $\tilde{U}_{1}^{0}$ and $\tilde{V}_{1}^{0}$ have the form

$$
\begin{gathered}
\tilde{U}_{1_{\alpha \beta}}^{0}=-i A_{\alpha} A_{\beta}^{*} \exp \left(\frac{i}{2}\left(u_{\alpha}-u_{\beta}\right) \xi+\frac{i}{2}\left(v_{\alpha}-v_{\beta}\right) \eta\right) \\
\tilde{V}_{1 \alpha \beta}^{0}=i B_{\alpha} B_{\beta}^{*} \exp \left(\frac{i}{2}\left(u_{\alpha}-u_{\beta}\right) \xi+\frac{i}{2}\left(v_{\alpha}-v_{\beta}\right) \eta\right)
\end{gathered}
$$

and depend on $\xi$ and $\eta$. The gauge transformation $\Psi^{0}=g \Phi^{0}$ with the function

$$
g_{\alpha \beta}=\delta_{\alpha \beta} \exp \left(\frac{i}{2} u_{\alpha} \xi+\frac{i}{2} v_{\alpha} \eta\right)
$$


transforms system $(1.31),(1.32)$ [with $\tilde{U}_{1}^{0} /(\lambda-1)$ and $\tilde{V}_{1}^{0} /(\lambda+1)$ are taken as $U_{1}$ and $\left.V_{1}\right]$, into a system with constant coefficients:

$$
\begin{aligned}
& \partial_{\xi} \Phi_{\alpha \beta}^{0}=-\frac{i}{2} u_{\alpha} \Phi_{\alpha \beta}^{0}-i \sum_{\gamma} \frac{A_{\alpha} A_{\gamma}^{*}}{\lambda-1} \Phi_{\gamma \beta}^{0}, \\
& \partial_{\eta} \Phi_{\alpha \beta}^{0}=-\frac{i}{2} v_{\alpha} \Phi_{\alpha \beta}^{0}+i \sum_{\gamma} \frac{B_{\alpha} B_{\gamma}^{*}}{\lambda+1} \Phi_{\gamma \beta}^{0} .
\end{aligned}
$$

Let us denote:

$$
\begin{array}{ll}
\left(U_{0}^{0}\right)_{\alpha \beta}=-\frac{i}{2} u_{\alpha} \delta_{\alpha \beta}, & \left(V_{0}^{0}\right)_{\alpha \beta}=-\frac{i}{2} v_{\alpha} \delta_{\alpha \beta} \\
\left(U_{1}^{0}\right)_{\alpha \beta}=-i A_{\alpha} A_{\beta}^{*}, & \left(V_{1}^{0}\right)_{\alpha \beta}=i B_{\alpha} B_{\beta}^{*} .
\end{array}
$$

The matrices constructed in such a way using relations (4.7) are the general solution of Eqs. (1.18), (1.19) to within a constant (independent of $\xi, \eta$ ) gauge rotation.

It is obvious that the solution of Eqs. (4.9), (4.10) can be found in a factorized form (1.23):

$$
\Phi_{\alpha \beta}^{0}=\sum_{\gamma} \Phi_{1_{\alpha \gamma}}^{0}(\xi, \lambda) \Phi_{2_{\gamma \beta}}^{0}(\eta, \lambda)
$$

the matrix functions $\Phi_{1_{\alpha \beta}}^{0}(\xi, \lambda)$ and $\Phi_{2_{\alpha \beta}}^{0}(\eta, \lambda)$ can be chosen unitary (for real $\lambda$ ) and commutative.

The procedure of finding such functions is a standard problem in the theory of linear differential equations with constant coefficients. Below we shall assume that the explicit form of the functions (4.11) is known.

3. In the previous section a scheme of constructing the function for a chiral field on a unitary group has been described. The explicit form of the functions $\Psi^{0}$ and $\Psi_{1,2}^{0}$ makes it possible to construct the function $X$ and to write out the $N$ soliton solution (2.1), (2.2) for Eq. (1). Here we present the simplest "single-soliton" solution. It should be recalled that it is defined by giving the complex parameter $\lambda_{0}$ and the projection operator $P_{0},\left(P_{0}^{2}=P_{0}, P_{0}^{\dagger}=P_{0}\right)$ in an $N$-dimensional unitary space. The projection operator can be easily written in a factorized form:

$$
P_{0}=X_{0}\left(X_{0}^{\dagger} X_{0}\right)^{-1} X_{0}^{\dagger},
$$

where $X_{0}$ is a rectangular matrix with $N$ lines and with $K$ columns, i.e., the matrix made up of $k$-linearly-independent complex vectors.

Suppose $\Psi^{0}(\xi, \eta, \lambda)$ is the vacuum wave function (of the first or second order). Now we write the projection operator depending on $\xi$ and $\eta$ :

$$
P(\xi, \eta)=X\left(X^{\dagger} X\right)^{-1} X^{\dagger},
$$

where

$$
X=\Psi^{0}\left(\xi, \eta \cdot \lambda_{0}\right) X_{0} .
$$


Now

$$
\begin{aligned}
& \varphi_{1}=\left(I+\frac{\lambda_{0}-\bar{\lambda}_{0}}{1+\bar{\lambda}_{0}} P(\xi, \eta)\right) \Psi_{2}^{0}(\eta,-1) \\
& \psi_{1}=\left(I-\frac{\lambda_{0}-\bar{\lambda}_{0}}{1-\bar{\lambda}_{0}} P(\xi, \eta)\right) \Psi_{1}^{0}(\xi, 1) .
\end{aligned}
$$

The first columns of the matrices $\varphi_{1}$ and $\psi_{1}$, as has been shown above, satisfy the system of Eqs. (1).

\section{The Gross-Neveu Model}

We consider now a model of fields on the real symplectic group $\operatorname{Sp}(2 N, \mathbb{R}) . U_{1}, V_{1}$ are the matrices of the form

$$
\left[\begin{array}{rr}
A & B \\
C & -A^{\mathrm{tr}}
\end{array}\right]
$$

where $A, B, C$ are the real $N \times N$ matrices, and $B^{\mathrm{tr}}=B, C^{\mathrm{tr}}=C$.

As in the previous case it is required that the rank of the matrices $U_{1}$ and $V_{1}$ should be unity. This can be achieved by putting $U_{1}^{0}=V_{1}^{0}$, and for these matrices $A=C=0$ :

$$
B=\left[\begin{array}{llll}
1 & & \\
& 0 & \\
& & \ddots & \\
& & & 0
\end{array}\right] .
$$

Consider the matrices $\varphi_{1}, \psi_{1}(2.1),(2.2)$. They can be chosen belonging to the symplectic group

$$
\psi_{1}^{-1}=-\mathscr{J} \psi_{1}^{\mathrm{tr}} \mathscr{J}, \quad \varphi_{1}^{-1}=-\mathscr{J} \varphi_{1}^{\mathrm{tr}} \mathscr{J},
$$

where $\mathscr{J}=\left(\begin{array}{rr}0 & -I \\ I & 0\end{array}\right)$ is a $2 N \times 2 N$ matrix. As in the previous section, we are mainly interested in the first column of the matrices $\psi_{1}$ and $\varphi_{1}$, since it is on its elements [taking into account (5.3)] that the non-linear Eqs. (2.3), (2.4) arise

$$
\begin{aligned}
& \partial_{\eta} \varphi_{1_{\alpha, 1}}=\frac{1}{2} \psi_{1_{\alpha 1}} \sum_{\beta=1}^{N}\left(\psi_{1_{\beta, 1}} \varphi_{1_{\beta+N, 1}}-\psi_{1_{\beta+N, 1}} \varphi_{1_{\beta, 1}}\right) \\
& \partial_{\xi} \psi_{1_{\alpha, 1}}=\frac{1}{2} \varphi_{1_{\alpha, 1}} \sum_{\beta=1}^{N}\left(\varphi_{1_{\beta, 1}} \psi_{1_{\beta+N, 1}}-\varphi_{1_{\beta+N, 1}} \psi_{1_{\beta, 1}}\right) .
\end{aligned}
$$

$\alpha=1,2, \ldots, 2 N$, the index should be understood $\bmod 2 N$.

The fields $\varphi_{1_{\alpha, 1}}$ and $\psi_{1_{\alpha, 1}}$ are a set of $2 N$ real fields. Two sets of $N$ complex fields can be introduced

$$
\psi^{\alpha}=\frac{1}{2}\left(\psi_{1_{\alpha, 1}}+i \psi_{1_{\alpha+N, 1}}\right), \quad \varphi^{\alpha}=-\frac{1}{2}\left(\varphi_{1_{\alpha, 1}}+i \varphi_{1_{\alpha+N, 1}}\right) .
$$

It can be verified by direct calculation that they satisfy the equations of the GrossNeveu model (2). 
The remaining columns satisfy the linear Dirac differential equations and do not impose any restrictions on the first column (cf. Paragraph 4).

2. Since the matrices $U_{1}^{0}$ and $V_{1}^{0}$ are nilpotent, the corresponding first order vacuum solution has no physical sense.

As in the previous section the vacuum solution is obtained directly from the system of Eqs. (2). It has the form:

$$
\psi^{\alpha}=\beta_{\alpha}^{1 / 2} A_{\alpha} e^{i \theta_{\alpha}}, \quad \varphi^{\alpha}=\beta_{\alpha}^{-1 / 2} A_{\alpha} e^{i \theta_{\alpha}},
$$

where

$$
\theta_{\alpha}=-\beta_{\alpha} \sigma_{0} \xi-\sigma_{0} \beta_{\alpha}^{-1} \eta+\theta_{\alpha}^{0}, \quad \sigma_{0}=\frac{1}{2} \sum_{\alpha=1}^{N} A_{\alpha}^{2}
$$

$A_{\alpha}, \beta_{\alpha}$ are arbitrary real constants. The matrices $\tilde{U}_{1}^{0}$ and $\tilde{V}_{1}^{0}$ for such a solution have the form

$$
\begin{aligned}
\tilde{U}_{1}^{0} & =\left[\begin{array}{lr}
A_{1} & B_{1} \\
C_{1} & -A_{1}^{\mathrm{tr}}
\end{array}\right], \quad \tilde{V}_{1}^{0}=\left[\begin{array}{lr}
A_{2} & B_{2} \\
C_{2} & -A_{2}^{\mathrm{tr}}
\end{array}\right] \\
A_{1 i j} & =-A_{i} A_{j}\left(\beta_{i} \beta_{j}\right)^{1 / 2} \sin \theta_{i} \cos \theta_{j} \\
B_{1 i j} & =-A_{i} A_{j}\left(\beta_{i} \beta_{j}\right)^{1 / 2} \sin \theta_{i} \sin \theta_{j} \\
C_{1 i j} & =A_{i} A_{j}\left(\beta_{i} \beta_{j}\right)^{1 / 2} \cos \theta_{i} \cos \theta_{j} \\
A_{2 i j} & =-A_{i} A_{j}\left(\beta_{i} \beta_{j}\right)^{-1 / 2} \sin \theta_{j} \cos \theta_{i} \\
B_{2 i j} & =A_{i} A_{j}\left(\beta_{i} \beta_{j}\right)^{-1 / 2} \cos \theta_{i} \cos \theta_{j} \\
C_{2 i j} & =-A_{i} A_{j}\left(\beta_{i} \beta_{j}\right)^{-1 / 2} \sin \theta_{i} \sin \theta_{j} .
\end{aligned}
$$

The gauge transformation $\Psi^{0}=g \Phi^{0}$, with the symplectic matrix $g$ of the following form

$$
g=\left[\begin{array}{ll}
\hat{g}_{11} & \hat{g}_{12} \\
\hat{g}_{21} & \hat{g}_{22}
\end{array}\right], \quad \begin{aligned}
& \left(\hat{g}_{11}\right)_{i j}=\left(\hat{g}_{22}\right)_{i j}=\delta_{i j} \sin \theta_{j} \\
& \left(\hat{g}_{12}\right)_{i j}=-\left(g_{21}\right)_{i j}=-\delta_{i j} \cos \theta_{j}
\end{aligned}
$$

transforms the system (1.31), (1.32), in which $U_{1}$ and $V_{1}$ are taken as $U_{1}^{0}$ and $V_{1}^{0}$, into the system:

$$
\begin{aligned}
& \partial_{\xi} \Phi^{0}=W_{1} \Phi^{0}, \\
& \partial_{\eta} \Phi^{0}=W_{2} \Phi^{0}
\end{aligned}
$$

with constant coefficients

$$
\begin{aligned}
W_{1,2} & =\left[\begin{array}{cc}
0 & w_{1,2} \\
\tilde{w}_{1,2} & 0
\end{array}\right], \quad \begin{array}{l}
\left(\tilde{w}_{1}\right)_{i j}=\sigma_{0} \beta_{i} \delta_{i j} \\
\left(w_{2}\right)_{i j}=-\sigma_{0} \beta_{i}^{-1} \delta_{i j}
\end{array} \\
\left(w_{1}\right)_{i j} & =-\sigma_{0} \beta_{i} \delta_{i j}-A_{i} A_{j}\left(\beta_{i} \beta_{j}\right)^{1 / 2}(\lambda-1) \\
\left(\tilde{w}_{2}\right)_{i j} & =\sigma_{0} \beta_{i}^{-1} \delta_{i j}-A_{i} A_{j}\left(\beta_{i} \beta_{j}\right)^{-1 / 2} /(\lambda+1) .
\end{aligned}
$$

According to their construction the matrices $W_{1}$ and $W_{2}$ commute at all values of the spectral parameter $\lambda$, therefore the solution of problem (5.9), (5.10) can be represented in the form $(1.23)$, the matrices $\Phi_{1}^{0}(\xi, \lambda)$ and $\Phi_{2}^{0}(\eta, \lambda)$ being symplectic and commutative. 
Below we assume the linear differential equations with constant coefficients (5.9), (5.10) to be solved and the functions $\Phi_{1}^{0}(\xi, \lambda), \Phi_{2}^{0}(\eta, \lambda)$, and, consequently, $\Psi^{0}(\xi, \eta, \lambda)$ to be known.

3. The problem of calculating soliton solutions, as is shown in Sect. 3, is reduced to the solution of a system of linear algebraic equations. Using the function $X$ (3.33) calculated in this Section we can write a soliton solution of the Gross-Neveu model:

$$
\begin{aligned}
\psi^{\alpha} & =\sum_{\beta, \gamma} X_{\alpha \beta}(\xi, \eta, 1) g_{\beta \gamma}(\xi, \eta)\left(\Phi_{1_{\gamma 1}}^{0}(\xi, 1)+i \Phi_{1_{\gamma+N, 1}^{0}}^{0}(\xi, 1)\right), \\
\varphi^{\alpha} & =\sum_{\beta, \gamma} X_{\alpha \beta}(\xi, \eta,-1) g_{\beta \gamma}(\xi, \eta)\left(\Phi_{2_{\gamma+N, 1}}^{0}(\eta,-1)-i \Phi_{2_{\gamma, 1}}^{0}(\eta,-1)\right) .
\end{aligned}
$$

\section{The Spinor Field Connected with the Orthogonal Group}

1. Suppose $G$ is the orthogonal group $O(N)$, and, consequently, $U_{1}$ and $V_{1}$ are real skew-symmetric matrices. Consider now the simplest case of matrices with lowest (non-zero) rank. In this case up to trivial transformations $U_{1}^{0}$ and $V_{1}^{0}$ have the form

$$
-U_{1}^{0}=V_{1}^{0}=\left[\begin{array}{cccc}
0 & \cdots & 0 & -1 \\
& 0 & & \\
1 & 0 & \cdots & 0
\end{array}\right] .
$$

The matrices $\varphi_{1}$ and $\psi_{1}$ (2.1), (2.2) can be chosen to belong to the orthogonal group $\left(\varphi_{1}^{\mathrm{tr}}=\varphi_{1}^{-1}, \psi_{1}^{\mathrm{tr}}=\psi_{1}^{-1}\right)$. The non-linear Eqs. (2.3), (2.4) arises for the first and last columns of the matrices $\varphi_{1}, \psi_{1}$ :

$$
\begin{aligned}
& \partial_{\eta} \varphi_{1_{\alpha, 1}}=-\frac{1}{2} \sum_{\beta}\left(\psi_{1_{\alpha, 1}} \psi_{1_{\beta, N}}-\psi_{1_{\alpha, N}} \psi_{1_{\beta, 1}}\right) \varphi_{1_{\beta, 1}} \\
& \partial_{\eta} \varphi_{1_{\alpha N}}=-\frac{1}{2} \sum_{\beta}\left(\psi_{1_{\alpha, 1}} \psi_{1_{\beta, N}}-\psi_{1_{\alpha, N}} \psi_{1_{\beta, 1}}\right) \varphi_{1_{\beta, N}} \\
& \partial_{\xi} \psi_{1_{\alpha, 1}}=-\frac{1}{2} \sum_{\beta}\left(\varphi_{1_{\alpha, 1}} \varphi_{1_{\beta, N}}-\varphi_{1_{\alpha, N}} \varphi_{1_{\beta, 1}}\right) \psi_{1_{\beta, 1}} \\
& \partial_{\xi} \psi_{1_{\alpha, N}}=-\frac{1}{2} \sum_{\beta}\left(\varphi_{1_{\alpha, 1}} \varphi_{1_{\beta, N}}-\varphi_{1_{\alpha, N}} \varphi_{1_{\beta, 1}}\right) \psi_{1_{\beta, N}}
\end{aligned}
$$

or passing to complex fields

$$
\psi^{\alpha}=\frac{1}{2}\left(\psi_{1_{\alpha, 1}}+i \psi_{1_{\alpha, N}}\right), \quad \varphi^{\alpha}=\frac{1}{2}\left(\varphi_{1_{\alpha, 1}}+i \varphi_{1_{\alpha, N}}\right)
$$

we get a system

$$
i \partial_{\xi} \psi^{\alpha}=\sum_{\beta} \mathscr{J}_{\alpha \beta}^{2} \psi^{\beta}, \quad i \partial_{\eta} \varphi^{\alpha}=\sum_{\beta} \mathscr{J}_{\alpha \beta}^{1} \varphi^{\beta},
$$

where

$$
\mathscr{J}_{\alpha \beta}^{1}=\psi^{\alpha} \psi^{* \beta}-\psi^{* \alpha} \psi^{\beta}, \quad \mathscr{J}_{\alpha \beta}^{2}=\varphi^{\alpha} \varphi^{* \beta}-\varphi^{* \alpha} \varphi^{\beta} .
$$

In accordance with (2.5) the action for this system has the form:

$$
S=\int d t d x\left[\sum_{\alpha}\left(i \psi^{* \alpha} \partial_{\xi} \psi^{\alpha}+i \varphi^{* \alpha} \partial_{\eta} \varphi^{\alpha}\right)-\frac{1}{2} \sum_{\alpha, \beta} \mathscr{J}_{\alpha \beta}^{1} \mathscr{J}_{\beta \alpha}^{2}\right],
$$

where $\mathscr{J}_{\alpha \beta}^{\mu}$ is the matrix of "color currents". 
2. Here, as well as in the unitary group, the first order solution can be taken as a vacuum solution.

It has the representation (1.24), where

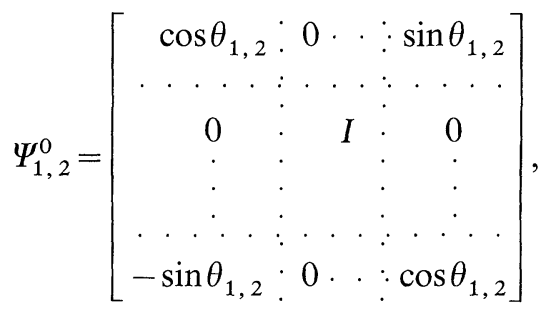

where $\theta_{1}=-\frac{\xi}{\lambda-1}, \theta_{2}=\frac{\eta}{\lambda+1}$; are constants.

The second-order vacuum solution can be obtained by the method described in Sect. 1 and illustrated in Sects. 4 and 5 on the example of the models (1) and (2).

3. The soliton solution on the background of the first-order vacuum for system (6.4) can be easily written down, using the function $X$ (3.33) calculated in Sect. 3 :

$$
\begin{aligned}
\psi^{\alpha} & =\sum_{\beta} X_{\alpha \beta}(\xi, \eta,-1)\left(\Psi_{2_{\beta 1}}^{0}(\eta,-1)+i \Psi_{2_{\beta, N}}^{0}(\eta,-1)\right) \\
\varphi^{\alpha} & =\sum_{\beta} X_{\alpha \beta}(\xi, \eta, 1)\left(\Psi_{1_{\beta, 1}}^{0}(\xi, 1)+i \Psi_{1_{\beta, N}}^{0}(\xi, 1)\right) .
\end{aligned}
$$

Acknowledgments. The authors are extremely grateful to Prof. J. Corones for his help in preparing the manuscript for publication.

\section{References}

1. Nambu, Y., Jona-Lasinio, G.: Phys. Rev. 122, 345 (1961)

2. Vaks, V.G., Larkin, A.I.: ZhETF 40, 282 (1961)

3. Gross, P.J., Neveu, A.: Phys. Rev. D 10, 3235 (1974)

4. Neveu, A., Papanicolaou, N.: Commun. Math. Phys. 58, 31 (1978)

5. Zakharov, V.E., Mikhailov, A.V.: ZhETF 74, 1953 (1978)

6. Zakharov, V.E., Takhtadyan, L.A.: TMF 38, 26 (1979)

7. Zakharov, V.E., Mikhailov, A.V.: Pis'ma v ZhETF 27, 47 (1978)

8. Zakharov, V.E., Shabat, A.B.: ZhETF 64, 1627 (1973)

9. Zakharov, V.E., Mikhailov, A.V.: Funct. Anal. Its Appl. 14, 55 (1980)

10. Kulish, P.P., Reiman, A.G. : Proc. Sci. Seminars. LOMI 77, 134 (1978)

11. Zakharov, V.E., Shabat, A.B.: Funct. Anal. Its Appl. 13, 13 (1979)

Communicated by Ya. Sinai

Received August 24, 1979 